\title{
Monotonicity in Time and Stationary Solutions for a Quasilinear Heat Equation with Source
}

\author{
Victor A. Galaktionov and Sergey a. Posashkov
}

\begin{abstract}
We consider the Cauchy problem for the quasilinear parabolic heat equation with source $u_{t}=\Delta u^{\sigma}+1+u^{\beta}$ in $R^{s} \times(0, T), \sigma>0 . \beta>I$ are fixed constants. with nonnegative bounded symmetric initial function. Two properties of monotone behaviours of the solution $u(|x|, t)$ for $x=0$ are investigated. 1. Monotonicity of large solutions: there exists a constant $M_{h}>0$ such that if $u\left(0, t_{0}\right) \geq M_{6}$ for some $t_{i j} \in[0, T)$, then $u_{t}(0, t) \geq 0$ for all $t \in\left[t_{0}, T\right)$. 2. $u(0 . t)$ does not decrease in $(0.7)$. It is shown that sufficient conditions for these propertics are quite different for five cases: i) $1<\beta<\sigma+1$, ii) $\beta=\sigma+1$, iii) $\sigma+1<\beta<\beta$, iv) $\beta=\beta$, v) $\beta>\beta$, where $\beta *=(\sigma+1)(N+2) /(N-2)$ for $N>2(\beta,=\infty$ for $N=1,2)$ is the critical Sobolev exponent.
\end{abstract}

\section{INTRODUCTION. MAIN RESULTS}

In this paper we consider the Cauchy problem for the quasilinear parabolic heat equation with source

$$
\begin{gathered}
u_{t}=\Delta u^{\sigma+1}+u^{\beta} \quad \text { in } \quad R^{N} \times(0,7) \\
u(x, 0)=u_{0}(x) \geq 0 \quad \text { in } \quad R^{N},
\end{gathered}
$$

where $\sigma>0$ and $\beta>1$ are fixed constants. Equation (1) is well-known equation arising in the theory and in different applications of the processes of the heat conduction and combustion in a medium, where the heat conductivity coefficient $k(u) \equiv(\sigma+1) u^{\prime}$ and the power of the energy emission $Q(u) \equiv u^{\beta}$ depend upon the temperature of the medium $u=u(x, 1) \geq 0$. 
We shall assume the initial function (2) satisfies the following hypotheses:

$$
\begin{aligned}
& u_{0}=u_{0}(r) \geq 0 \text { in } R^{N}, r=|x|: M_{1}=\sup u_{0}<\infty: \\
& u_{0}(r) \text { is the continuous function in } R_{4}^{\prime}=(0, \infty) .
\end{aligned}
$$

$$
\left(u_{0}^{(1)} u u_{0}\right)(0)=0 .
$$

Under these hypotheses, there exists the unique weak local (in time) solution $u=u(r, t)$ of the problem (1), (2), which is nonnegative continuous function in $R^{*} \times(0, T)$, where $T \in(0, \infty]$ is il linite or an infinite existence time. See a full list of references given in [11]. Notice that $u(r, t)$ is the classical solution at any point $(r, t)$ where $u(r, t)$ is strictly positive.

The main results of the paper are devoted to the analysis of the behaviour with time of the temperature at the single point $x=0$. This yields the conditions on the initial temperature for the ignirion of the combustion process at the single point $x=0$.

We shall consider two types of the monotone behaviours of $u(0, t)$.

Property (ML) (Monotoniciry of the Large solution): there exists a constant $M_{h}>0$, depending on the initial function, such that if $u\left(0, t_{0}\right) \geq M_{k}$ for some $t_{0} \in[0, T)$, then

$$
u_{f}(0, t) \geq 0 \text { for all } t \in\left[i_{1}, 7\right)
$$

Property (M) (Monotonicity for arbitrary l):

$$
u(0, t) \text { doesn't decrease in }(0,7) \text {. }
$$

We show that above properties (ML) and (M) depend on the initial function $\iota_{0}$, the dimension of the space $N \geq 1$ and exponents $\sigma, \beta$ of equation (1). These properties are quite different for live cases: i) $1<\beta<\sigma+1$, ii) $\beta=\sigma+1$, iii) $\sigma+1<\beta<\beta_{*}$, iv) $\beta=\beta_{*}$, v) $\beta>\beta_{*}$, where

$$
\beta_{*}=(\sigma+1)(N+2) /(N-2) \text { for } N>2\left(\beta_{*}=\infty \text { for } N=1,2\right)
$$

is the critical Sobolev exponent for the nonlinear elliptic operator in the, righthand side of (1).

Let $B_{\varepsilon}=\{r<\varepsilon\}$ be the ball in $R^{x}$ of a radius $\varepsilon>0$ with the boundary $S_{x}=\{r=\varepsilon\} . D_{\varepsilon}=R^{N} \backslash B_{\varepsilon}$ and denote

$$
h^{*}(r)=\left(* r^{-(x-2)} 2(n+1) \text { for } r>0\right. \text {. }
$$




$$
\begin{gathered}
c *=\left[\frac{N(N-2)}{4}\right]^{(N-2) 4(\sigma+1)}(N>2) ; \\
h(r)=c r^{-2,[\beta-(\sigma+1)]} \text { for } r>0, \\
c=\left[\frac{\left.-\frac{2 N(\sigma+1)}{\beta-(\sigma+1)}\right]^{1 /[\beta-(\sigma+1)]}(\beta>\sigma+1) ;}{h_{\infty}(r)=c_{\infty} r^{-2 ;[\beta-(\sigma+1)]} \text { for } r>0,}\right. \\
=\left\{\frac{2(\sigma+1)[\beta(N-2)-N(\sigma+1)]}{[\beta-(\sigma+1)]^{2}}\right]^{-1 /[\beta-(\sigma+1)]}, \\
(\beta>(\sigma+1) N /(N-2) \text { for } N>2) .
\end{gathered}
$$

We now state the main results of the paper.

Theorem $1(I<\beta<\sigma+I)$. Let $I<\beta<\sigma+I$ and let (3) holds. Then

(i) If $u_{0}(r)$ satisfies

there exists a large constant $R>0$ such that $u_{0}^{o+1}$ is

uniformly Lipschitz continuous in $D_{k}$,

then (ML) holds.

(ii) If $u_{0}^{g+i}(r) \in C^{\prime}$ and

$$
\left|\left[u_{0}^{\sigma+1}(r)\right]^{\prime}\right|=o(r(\beta+\sigma+1)(\alpha+1-\beta)) \text { as } r \rightarrow+\infty,
$$

then (ML) holds.

(iii) If $u_{0}^{\sigma+l}(r) \in C^{l}$ and

$$
\left(u_{0}^{a+1}(r)\right)+u_{0}^{\beta}(r) r / N>0 \text { in }\{r>0\} \cap\left\{u_{0}>0\right\}
$$

then (M) holds.

Theorem 2 $(\beta=\sigma+1)$. Let $\beta=\sigma+1$ and let (3) holds. Then

(i) If $u_{i}(r)$ sativfies 
where $z_{1}>0$ is the first zero of Bessel function $J_{(N-2,3}$, then $(M L)$ holds.

(ii) If $u_{0}^{(+1}(r) \in C^{\prime}$ and

$$
\left(u_{0}^{\sigma+l}(r)\right)^{r}+u_{0}^{\mathrm{o}+l}(r) r / N>0 \text { in }\left\{0<r<z_{N}\right\} \cap\left\{u_{0}>0\right\} .
$$

then (M) holds.

(iii) If $u_{0}(r)$ is the nondecreasing function in $\left\{0<r<z_{N}\right\}$, then (M) holds.

Theorem $3\left(\sigma+I<\beta<\beta_{*}\right)$. Let $\sigma+I<\beta<\beta_{*}$ and let $(3)$ holds. Then

(i) If for some small $\varepsilon>0$

$$
u_{0}^{\sigma+t}(v) \text { is Lipschitz continuous in } B_{i}
$$

then (ML) holds,

(ii) If $u_{0}^{\text {g+t }}(r) \in C^{l}$ and

$$
\left(u_{0}^{\mathrm{g}+1}(r)\right)^{r}+u_{k}^{\beta}(r) r / N>0 \text { in }\{r>0\} \cap\left\{u_{0}>0\right\} .
$$

then (M) holds.

(iii) If $u_{b}(r)$ is the nondecreasing function in $\left\{r<l_{\theta}\right\}$, where

$$
l_{0}=c_{1}\left(u_{0}(0)\right)^{-1 \beta-10+1) / 2}
$$

and $c_{l}=c_{1}(\sigma, \beta, N)$ is some positive constant, then (M) holds.

(iv) If $u_{t}(r)$ is the nondecreasing function in $\left\{r>0 \mid u_{i j}(r)<h(r)\right\}$, then (M) holds.

(v) Let $l_{*}=\sup \left\{a>0 \mid u_{f}(r)\right.$ is the nondecreasing function for $\left.r \in(0, a)\right\}>0$. Then (ML) holds for

$$
M_{h_{1}}=\left((c / /)_{*}\right)^{-/|\beta-(\alpha+1)|} .
$$


Theorem $4\left(\beta=\beta_{*}\right)$. Let $\beta=\beta_{*}$ for $N \geq 3$ and let (3) holds. Then

(i) If $u_{0}(r)$ satisfies (13) for some $c>0$ and

$$
u_{0}(r)>0 \text { for } a / l r>0
$$

there exists constant $m_{0}>0$ such that

$$
u_{0}(r)>m_{0} \cdot r^{-(N-?) /(\sigma+1)} \text { for any large } r>0 \text {, }
$$

then (ML) holds.

(ii) Assertion (ii) of Theorem 3 is valid.

(iii) If $u_{0}(r)$ is the nondecreasing function in $\left\{r>0 \mid u_{0}(r)<h^{*}(r)\right\}$, then (M) holds.

Theorem $5\left(\beta>\beta_{*}\right)$. Let $\beta>\beta_{*}$ for $N \geq 3$ and led (3) holds. Then

(i) Let $u_{0}(r)=h_{\infty}(r)$ for a unique point $r=r_{*}>0, u_{0} \in C^{\prime}$ in a small neighbourhood of $r=r_{*}$ and $u_{0}\left(r_{*}\right)>h_{s_{0}}(r *)$. Let for any sufficiently small $\varepsilon>0$ the set $\left\{r>0|| u_{0}(r) / h_{\infty}(r)-1 \mid<\varepsilon\right\}$ be a finite connected interval containing the point $r=r_{*}$. Then (ML) holds.

(ii) Assertion (ii) of Theorem 3 is valid.

(iii) If $u_{0}(r)$ is the nondecreasing function in $\left\{r>0 \mid u_{0}(r)<h(r)\right\}$, then (M) holds.

Proofs of Theorems $1-5$ are based on intersection comparison of the solution $u(r, t)$ with the set of the stationary solutions $\{U\}$. Therefore, we begin with the analysis of the set $\{U\}$.

\section{SOME PROPERTIES OF THE SET OF THE STATIONARY SOLUTIONS}

We now describe some well-known properties of the stationary solutions $U(r ; \lambda)$ satisfying

$$
\begin{gathered}
\Delta U^{0+1}+U^{\beta} \equiv r^{1-N}\left(r^{\prime-1}\left(U^{0+1}\right)_{r}\right)_{r}+\dot{U}^{\beta}=0 \text { for } r>0, \\
U(0 ; \lambda)=\lambda>0, \\
U_{r}(0 ; \lambda)=0,
\end{gathered}
$$

where $\lambda>0$ is an arbitrary fixed constant. 
One can see that equation (17) is invariant under the sell-similar transformation and hence

$$
U(r ; \lambda)=\lambda U\left(r \lambda^{\prime \prime} ; 1\right), m=[\beta-(\sigma+1)] / 2 \text {, for any fixed } \lambda>0 .
$$

It is well-known (see e.g. [10]) that for $\beta \geq \beta$, any solution of (17) is strictly positive for all $r>0$ and $U(\infty: \lambda)=0$. For $\mathrm{I}<\beta<\beta_{*}$ the function $U(r ; \lambda)$ vanishes at some point $r=l(\lambda)$, where

$$
l(\lambda)=c_{1} \cdot \lambda^{-m}
$$

$c_{1}(\sigma, \beta, N) \equiv /(1)>0$ is given in (15). In this case we let $U(r ; \lambda) \equiv 0$ for $r>l(\lambda)$. For $\beta \geq \beta_{*}$ we formally let $l(\lambda)=+\infty$.

Thus, for $\beta>1$ we have introduced one parametric set of the stationary solutions $\{U(r ; \lambda) ; \lambda \geq 0\}(U(r ; 0) \equiv=0)$. In all cases for arbitrary fixed $\lambda>0$ $U(r ; \lambda)$ is the monotone decreasing function in the domain of positivity. "The function $U(r ; \lambda)$ is the continuous function with respect to $r$ and $\lambda$. From well-known properties of solution of the Cauchy problem for the ordinary differential equation (17) we get that there exists the continuous dependence on $\lambda$ of the solution $U(r ; \lambda)$ in any compact from $R_{+}^{1}$ and the derivative $\left(U^{\alpha+1}\right)$, in any compact from the domain $\omega(\lambda)=[0, /(\lambda))$ of positivity of the stationary solution.

The case $\beta \in(1, \sigma+1)$. For $\beta \in(1, \sigma+1)$ from (19) it follows that

$$
l(\lambda) \rightarrow+\infty \text { monotone as } \lambda \rightarrow+\infty \text {. }
$$

ldentity (18) yields the following conditions

$$
\begin{gathered}
U(r ; \lambda) \rightarrow+\infty \text { uniformly in }[0, / / 2] \text { as } \lambda \rightarrow+\infty \\
\left(U^{\circ+1}\right),(r ; \lambda) \rightarrow-\infty \text { uniformly in }[/ / 2, /] \text { as } \lambda \rightarrow+\infty \text {. }
\end{gathered}
$$

Introduce for a fixed constant $M>0$ the set $g(\lambda, M)=\{r>0\}$

$0<U(1 ; \lambda)<M\}$. From (18). (19) one can obtain the following estimates: if $r \in g(\lambda, M)$ then

$$
\begin{gathered}
r=/(\lambda)(1+o(1)) \rightarrow \infty \text { as } \lambda \rightarrow \infty . \\
\left|\left(U^{\alpha+1}\right),(r, \lambda)\right|=k, r^{-(\beta+o+1) 2 m}(1+o(1)) \rightarrow \infty \text { as } \lambda \rightarrow \infty,
\end{gathered}
$$

where $k_{1}=k_{1}(\sigma, \beta, N)>0$ is some constant.

The case $\beta=\sigma+l$. In this case equation (17) has the explicit solution

$$
U(r ; \lambda) \equiv U_{s}(r ; \lambda)=\lambda\left\{\Gamma^{\top}(N / 2) 2^{r} r^{-n} J_{u}(r)\right\}, r \in\left[0, z_{N}\right)
$$


where $\nu=(N-2) / 2, \mathrm{~J}^{\prime}(N / 2)$ is Euler's Gamma function, $z_{N^{\prime}}$ is the first zero of Bessel function $J_{v}(r)\left(U\left(z_{,}, \lambda\right)=0\right)$.

For any fixed arbitrary small $\varepsilon>0$

$$
\begin{gathered}
U_{S} \rightarrow+\infty \text { uniformly in } r \in\left[0, z_{N}-\varepsilon\right] \text { as } \lambda \rightarrow+\infty, \\
\left(U_{S}^{0+1}\right), \rightarrow-\infty \text { uniformly in } r \in\left[z_{N}-\varepsilon, z_{N}\right) \text { as } \lambda \rightarrow+\infty .
\end{gathered}
$$

The case $\beta \in\left(\sigma+l, \beta_{*}\right)$. Then it follows from (19) that

$$
l(\lambda) \rightarrow 0 \text { monotone as } \lambda \rightarrow+\infty .
$$

and properties (21), (22) are also valid.

The case $\beta=\beta_{*}(N \geq 3)$. It is well-known [12] that for $\beta=\beta_{*}, N \geq 3$, equation (17) admits the explicit strictly positive solution

$$
U(r ; \lambda)=\lambda \cdot\left[\frac{N(N-2)}{N(N-2)+\lambda^{4(\sigma+1)(N-2) r^{2}}}\right]^{(N-2) 2(a+1)}
$$

for $r>0$. It has the following properties:

$$
U(r ; \lambda)=c_{2} \lambda^{-1} r^{-(N-2)(o+1)}(1+o(1)) \text { as } r \rightarrow \infty .
$$

where $c_{2}=[N(N-2)]^{(N-2) 2(a+1)}$;

$U(r ; \lambda) \rightarrow 0$ uniformly in any set $[\delta,+\infty)$ as $\lambda \rightarrow \infty$,

$$
\begin{gathered}
\left(U^{v+1}\right)_{r}(r ; \lambda)--\infty \text { uniformly in any set } \\
\left\{r>0 \mid m_{1} \leq U(r ; \lambda) \leq m_{2}\right\} \text { as } \lambda \rightarrow \infty,
\end{gathered}
$$

where $\delta, m_{1}<m_{2}$ are arbitrary fixed positive constants.

The case $\beta>\beta_{*}, N \geq 3$. Then $U(r ; \lambda)>0$ for any $r>0, U(+\infty: \lambda)=0$ and there hold $[10]$

$$
\begin{gathered}
U(r ; \lambda)=h_{\infty}(r)(1+o(1)), \\
U_{r}(r ; \lambda)=h_{\infty}^{\prime}(r)(1+o(1)) \text { as } r \rightarrow+\infty,
\end{gathered}
$$

where the function $h_{\infty}(r)$ is given by (7). From (18), (30) one can easily verify that for any fixed $\delta>0$

$$
U(r ; \lambda) / h_{\infty}(r)-1
$$




$$
U_{r}(r ; \lambda) / h_{\infty}^{\prime}(r)-1 \text { uniformly in any set }[\delta,+\infty) \text { as } \lambda \rightarrow \infty \text {. }
$$

The estimates of $U(r ; \lambda)$. The first estimate holds for arbitrary $\beta>1$. By using monotonicity of the function $U(r ; \lambda)$ for $r>0$ we obtain the following inéquality

$$
r^{1-N}\left(r^{N-1}\left(U^{a+1}\right)_{r}\right)_{r}=-U^{\beta} \geq-\lambda^{\beta} \text { for } r>0 .
$$

Integrating twice this inequality yields the lower estimate

$$
U(r ; \lambda) \geq U_{-}(r ; \lambda) \equiv \lambda\left(1-r^{2} / r_{0}^{2}\right)_{+}^{1,(s+1)} \text { for } r>0,
$$

where $r_{0}=r_{0}(\lambda) \equiv(2 N)^{12} \cdot \lambda-[\beta-(\omega+1)] \cdot 2$.

By integrating equation (17) and by using the monotonicity of the function $U(r ; \lambda)$ for $r>0$ we get the following inequality:

$$
\left(U^{a+1}\right)_{r}+U^{\beta} \frac{r}{N} \leq 0 \text { for } r>0 .
$$

The second estimate for $\beta \in(\sigma+1,+\infty)$ we shall derive by integrating (33). Then we have for $r>0$

$$
U(r ; \lambda) \leq\left[\lambda-[\beta-(\sigma+1)]+\frac{r^{2}[\beta-(\sigma+1)]}{2 N(\sigma+1)}\right]^{-1 /[\beta-(\sigma+1) \mid},
$$

and hence

$$
U(r: \lambda) \leq h(r), r>0, \text { for any fixed } \lambda>0,
$$

where $h(r)$ is given in $(7)$.

The tangent curve. Upper estimate (34) implies that for any $\beta>\sigma+$ I there exists the tangent curve $L=L(r)$ of the set $\{U(r ; \lambda)\}$ and the following upper estimate

$$
L(r) \equiv \sup _{\lambda>0} U(r ; \lambda) \leq h(r) \text { for } r>0
$$

holds. Moreover, from (32) for $\beta>\sigma+1$ we obtain the lower estimate of $L(r)$ :

$$
L(r) \geq L_{-}(r) \equiv \sup _{\lambda>0} U_{-}(r ; \lambda)=c_{3} r^{-2}[\beta-(s+1)] \text { for } r>0,
$$

where

$$
C_{3}=\left[\frac{\beta-(\sigma+1)}{\beta}\right]^{\mid(\sigma \div 1)}\left[\frac{2 N(\sigma+1)}{\beta}\right]^{\mid[\beta-(\sigma+1)]}
$$


For $\beta=\beta$, the tangent curve can be calculated explicitly:

$$
L(r)=h^{*}(r) \text { for } r>0,
$$

where the function $h^{*}(r)$ is determined by $(7)$.

\section{INTERSECTION COMPARISON}

1. Let $\omega(\lambda)=[0, /(\lambda))$ be the domain of positivity of the function $U(r ; \lambda)$ for fixed $\lambda>0\left(\omega(\lambda) \equiv[0,+\infty)\right.$ for $\left.\beta \geq \beta_{*}\right)$. Let $N(r ; \lambda)$ for lixed $t \in[0, T)$ be the number of intersections of the functions $u(r, t)$ and $U(r, \lambda)$ in $\omega(\lambda)$ (see e.g. [6]-[8], [15]) or, which is the same, the number of sign changes of the difference $u(r, t)-U(r ; \lambda)[1],[14],[16]$. This implies that the functions $u(r, t)$ and $U(r, \lambda)$ are positive in a small neighbourhood of any intersection. Since the solutions $u(r, t)$ and $U(r ; \lambda)$ are classical there, each intersection is an isolated point for any fixed $t>0$ [1], [13], [16]. Without loss of generality we shall assume that for $t=0$ and for all $\lambda>0$ there exist only points of intersection, and $N(0 ; \lambda)<+\infty$.

The following Lemma $l$ is well-known for classical solutions, see [1], [6], [7], [13]-[16]: for weak solutions of degenerate equations see results in [8], [15].

Lemma 1. Fix arbitrary $\lambda>0$. Let (3) holds and $N(0 ; \lambda)<\infty$. Then $N(t ; \lambda) \leq N(0 ; \lambda)+$ l. If $N(0 ; \lambda) \leq 1$ for some fixed $\lambda>u_{0}(0)$. then

$$
N(t ; \lambda) \leq l \text { for any } t \in(0, T) .
$$

Proof. Below we use the standard technique of construction of the weak solution of the Cauchy problem (1), (2) [II]. Fix an arbitrary small $\varepsilon>0$ and denote

$$
u_{0 \varepsilon}(x) \equiv \max \left\{u_{0}(r), \varepsilon\right\}>0 \text { for } r \geq 0
$$

Clearly, $u_{0 \varepsilon}(x) \rightarrow u_{0}(r)$ as $\varepsilon \rightarrow 0$ uniformly in $R^{N}$. The Cauchy problem for (1) with initial function (38) has the unique classical strictly positive solution $u_{\varepsilon}(r, t) \geq \varepsilon$ in $R^{N} \times(0, T)$ [3]. Moreover (see [11] and references therein)

$$
u_{\varepsilon}(r, t) \rightarrow u(r, t) \text { as } \varepsilon \rightarrow 0
$$

uniformly in any compact set from $R^{N} \times(0, T)$. 
Let $N_{x}(t ; \lambda)$ for fixed $t \in[0,7)$ be the number of the points of intersection of the solutions $u_{\varepsilon}(r, t)$ and $U(r ; \lambda)$ in $\omega(\lambda)$. One can see that $N_{k}(0 ; \lambda) \leq$ $N(0 ; \lambda)+1$ (one additional point of intersection agivens in the right-hand side of the last inequality can arise on the boundary $r=l(\lambda)$ of $\omega(\lambda))$. Consider for arbitrary fixed $t \in(0,7)$ the domain $\Omega(t)=\omega(\lambda) \times(0, t)$. It is well-know that $N_{\mathrm{c}}(t ; \lambda)$ is not greater than the number of the sign changes of the difference $w_{\varepsilon}(r, t ; \lambda) \equiv u_{\varepsilon}(r, t)-U(r ; \lambda)$ on the parabolic boundary of $\Omega(t)$ $[1],[13],[14],[16]$. Since $u_{c}(l(\lambda), t)>0$ for all $t \in(0,7)$ we get the inequality

$$
N_{\varepsilon}(t ; \lambda) \leq N_{\varepsilon}(0 ; \lambda) \text { for } t \in(0, T)
$$

From (39) by using the continuity of $u(r, t)$ we can conclude that

$$
N(r ; \lambda) \leq N_{\varepsilon}(t ; \lambda) \text { for any small } \varepsilon>0,
$$

and hence $N(t ; \lambda) \leq N(0 ; \lambda)+1$.

If $\lambda>u_{0}(0)$ and $N(0 ; \lambda)=0$ or 1 , then it is easily seen that $N_{\mathrm{f}}(0 ; \lambda) \leq 1$ for any small $\varepsilon>0$. Then in both cases we have $N(t ; \lambda) \leq \mathrm{I}$ for all $t \in(0,7)$, which completes the proof.

2. Comparison with the set of the stationary solutions. "The following two Lemmas 2, 3 are based on the analysis of the number of intersection of the initial function $u_{0}(r)$ with the set $\{U(r, \lambda)\}$ of the stationary solutions.

These I.emmas yield the sufficient conditions for the properties (ML) and (M).

Lemma 2 (property (ML))). Let (3) holds and there erists $\lambda_{*}>M_{1}$ such that

$$
N(0 ; \lambda) \leq l \text { for } a / / \lambda>\lambda_{*} \text {. }
$$

Then (ML) holds with $M_{k}=\lambda_{*}$.

Lemma 3 (property (M)). Let (3) holds and

$$
\begin{gathered}
N(0 ; \lambda) \leq l \text { for all } \lambda>\lambda_{\theta}=u_{f(}(0), \\
u_{i l}(r) \geq U\left(r ; \lambda_{i t}\right) \text { in } R_{f}^{\prime} .
\end{gathered}
$$

Then (M) holds. 
Proof of Lemma 2. Let $u_{0} \notin\{U(r ; \lambda)\}$ and (40) is valid for some $\lambda_{\text {s. }}$. Fix arbitrary $\lambda>\lambda_{\text {. }}$. Let $u\left(0, t_{\lambda}\right)=\lambda$ for some $t_{\lambda} \in(0, T)$ such that $u(0, t)<\lambda$ for all $t \in\left(0, t_{\Lambda}\right)$. We shall show that (see also [4], [15, Chapter IV])

$$
u\left(r, t_{\lambda}\right) \geq U(r ; \lambda) \text { for all } r \geq 0 \text {. }
$$

Suppose (42) is not valid. Then from (40) and Lemma 1 it follows that $N\left(t_{\lambda} ; \lambda\right) \leq 1$.

Consider the first case when $N\left(t_{\lambda} ; \lambda\right)=0$. Then $u\left(r, t_{\lambda}\right) \leq U(r ; \lambda)$ in $\omega(\lambda)$. Hence, without loss of generality we may assume supp $u\left(r, t_{\lambda}\right) \subseteq \omega(\lambda)$. Since by well-known properties of the interface [1I] supp $u_{0} \subseteq \operatorname{supp} u\left(r, t_{\Lambda}\right)$ and hence by Lemma I we have $N(0 ; \lambda)=0$. Therefore. $u_{0}(r) \leq U(r ; \lambda)$ in $\omega(\lambda)$ and $u(l(\lambda), t)=0$ for $t \in\left[0, t_{\lambda}\right]$. Then by the strong maximum principle [3. Chapter II], applied for $u(r, t)$ in the domain of positivity near the origin, we get $u\left(0, t_{\lambda}\right)<U(0 ; \lambda)$, whence the contradiction.

Consider the second case when $N\left(t_{\lambda} ; \lambda\right)=I$, i.e. there exists one sign change of the difference $w\left(r, t_{\lambda} ; \lambda\right) \equiv u\left(r, t_{\lambda}\right)-U(r ; \lambda)$ in $\omega(\lambda)$. Then by using the continuity of $U(r ; \lambda)$ with respect to $\lambda$ we get that there exists some sufficiently small $|\varepsilon|>0, \lambda+\varepsilon>\lambda_{*}$, such that the difference $w\left(r, t_{\lambda} ; \lambda\right)$ has at least two sign changes in $\omega(\lambda+\varepsilon)$ and $N\left(t_{\lambda} ; \lambda+\varepsilon\right) \geq 2$. See the similar analysis in [6-8], [15, p. 384]. This contradicts (37) and (40).

Thus, (42) is valid. Then by the comparison theorem $u(r, t) \geq U(r ; \lambda)$ in $\omega(\lambda)$ for all,$\in\left(t_{\lambda}, T\right)$. Since $u\left(0, t_{\lambda}\right)=\lambda=U(0 ; \lambda)>0$ and $u(0, t) \geq \lambda$ for any $t \in\left(t_{\lambda}, 7\right)$ we obtain the inequality $u_{,}\left(0, t_{\lambda}\right) \geq 0$ and hence $(\mathrm{ML})$ is valid because $\lambda>\lambda_{*}$ is arbitrary.

Proof of Lemma 3. Since $u_{0}(r) \geq U\left(r ; \lambda_{0}\right)$ in $R_{+}^{l}$ by the comparison theorem $u(r, t) \geq U\left(r ; \lambda_{0}\right)$ in $R_{+}^{1} \times(0, T)$. This implies that $u(r, t)$ doesn't decrease with time for $t=0$ at any point $r=r_{*}$, where $u_{0}\left(r_{*}\right)=U\left(r_{*} ; \lambda_{0}\right)>0$, in particular, at the point $r=0$. The end of the proof is quite similar to the proof of Lemma 2 with $\lambda_{*}=\lambda_{0}$.

\section{PROOFS OF THEOREMS 1-5}

Proofs of Theorems 1-5 are based on the properties of the set of the stationary solution and on Lemmas 2 and 3.

Proof of Theorem 1. (i) From (20)-(22) and (8) it follows that there exists some sufficiently large $\lambda_{*}=\lambda_{*}(R)>0$ such that $N(0, \lambda) \leq 1$ for all $\lambda>\lambda$. Then by Lemma 2 (i) holds. 
Notice that, roughly speaking, for $\beta \in(1, \sigma+I)$ the property (ML) doesn't depend on the behaviour of the initial function $u_{0}(r)$ in a neighbourhood of origin.

(ii) Estimates (22) with $M=M_{1}$ (see (3)) and the condition (9) imply that for any sufficiently large $\lambda>0$ the inequality $N(0 ; \lambda) \leq 1$ holds. Hence by Lemma 2 (ii) is valid.

(iii) From estimate (33) and condition (10) we get the ordinary differential inequality of the first order for the dilference $z(r)=u_{0}(r)-U\left(r ; \lambda_{0}\right)$. $\lambda_{0}=u_{0}(0)>0$;

$$
(a(r) z)+b(r) z>0 \text { in } \omega(\lambda) \cap\left\{u_{0}>0\right\}, z(0)=0
$$

where $a(r)$ and $b(r)$ are some smooth nonnegative functions. Then by the comparison theorem $z \geq 0$ in $\omega(\lambda) \cap\left\{u_{0}>0\right\}$ and hence $u_{0}(r) \geq U\left(r ; \lambda_{0}\right)$ in $R_{+}^{1}$ Moreover, if for $\lambda>\lambda_{0}$ there cxists some point of intersection $r=r_{*}$ of the functions $u_{0}(r)$ and $U(r ; \lambda)$ in $\omega(\lambda)$, then $\left(u_{0}\right)_{r}\left(r_{*}\right)>U_{r}\left(r_{*} ; \lambda\right)$. This implies that $u_{0}(r) \geq U(r ; \lambda)$ for all $r>r_{*}$ and hence $N(0 ; \lambda) \leq 1$. Then by Lemma 3 (iii) is valid.

From (i) we have

Corollary 1. Let $\beta \in(I, \sigma+l)$. (3) holds and let $u_{0}(r)$ be a compachly supported function. Then (ML) holds.

Proof of Theorem 2. (i) This is similar to the proof of (i) of Theorem $I$. Explicit solution (23) and properties given in (24), (25) are used.

Note that for $\beta=\sigma+1$ the property (ML) at the origin depends on the behaviour of $u_{0}$ in a small left neighbourhood of the point $r=z_{x^{\prime}}$.

(ii) See the proof of (iii) of Theorem 1.

(iii) Denote $\lambda_{0}=u_{0}(0)$. Then $u_{0}(r)>U\left(r ; \lambda_{0}\right) \equiv U_{S}\left(r ; \lambda_{0}\right)$ for all $r \in\left(0, z_{i}\right)$ since $u_{0}(r)$ is nondecreasing function in $\left(0, z_{N}\right)$. Since $U_{r}(r ; \lambda)<0, r \in\left(0, z_{N}\right)$. for any $\lambda>\lambda_{0}$ and $U(r ; \lambda) \equiv 0$ for $r \geq z_{i}$, we get $N(0 ; \lambda)=1$ and lemma 3 can be used.

The assertion (i) yiclds

Corollary 2. Let $\beta=\sigma+1$. (3) holds and let $u_{i}(r)$ be a compactly. suppored finction swh that $s u p\left\{r>0 \mid u_{0}(r)>0\right\}<z_{x}$. Then $(M L)$ is valid. 
Proof of Theorem 3. (i) Properties (21), (22), (26) and the condition (13) guarantee the inequality $N(0 ; \lambda) \leq 1$ for all sufficiently large $\lambda>0$. Hence Lemma 2 can be applied. Therefore, the property (ML) depends on the behaviour of the initial function near $r=0$.

(ii) See the proof of (iii) of Theorem 1 .

(iii) Since $U_{r}(r ; \lambda)<0$ in $\omega(\lambda)$, which is the domain of positivity of the function $U(r ; \lambda)$, from $(15)$, (18), (19) we get that $u_{0}(0)=U\left(0 ; u_{0}(0)\right)$ and $u_{0}(r)>U\left(r ; u_{0}(0)\right)$ for $r \in\left(0, l_{0}\right)$. From $(26)$ we obtain the inequality $N(0 ; \lambda)=1$ for all $\lambda>u_{0}(0)$. Now one can use I emma 3 .

(iv) Since $h(r) \geq \sup _{\lambda>0} U(r ; \lambda)(\sec (35))$ and $u_{0}(r)$ is nondecreasing function in $\left\{r>0 \mid u_{0}(r)<h(r)\right\}$ we get that $u_{0}(r) \geq U\left(r ; u_{0}(0)\right)$ for all $r>0$ and $N(0 ; \lambda)=1$ for all $\lambda>u_{0}(0)$. Then by Lemma 3 (iv) holds.

(v) See (19), (26) and the proof of (iii) of this Theorem.

Proof of Theorem 4. (i) Choose $\delta=\varepsilon(\sec (13))$. Then from (13) and (29) for all sufficiently large $\lambda>0$ there exists unique intersection of the functions $u_{0}(r)$ and $U(r ; \lambda)$ in $(0, \delta)$. By using $(16),\left(16^{\prime}\right),(28),(29)$ we get that there are no points of intersection in $[\delta, \infty)$, i.e. $N(0 ; \lambda)=1$ for large $\lambda>0$. Hence, (ML) holds.

(ii) See the proof of assertion (iii) of Theorem 1. Notice that for $\beta \geq \beta$, under hypothesis (14) the initial function should be strictly positive.

(iii) See the proof of (iv) of Theorem 3.

Proof of Theorem 5. (i) Fix small $\delta>0$ such that $h_{\infty}(\delta)>2 M_{1}$. By using (31) for any large $\lambda>0$ we can choose sufficiently small $\varepsilon>0$ such that

$$
\left|U(r ; \lambda) / h_{\infty}(r)-1\right|<\varepsilon, \quad\left|U_{r}(r ; \lambda) / h_{\infty}^{\infty}(r)-1\right|<\varepsilon
$$

in $[\delta, \infty)$. Since $u_{0} \in C^{l}$ in the neighbourhood of unique point $r=r_{*}$. where $u_{0}\left(r_{*}\right)=h_{\infty}\left(r_{*}\right)$ and $u_{0}^{\prime}\left(r_{*}\right)>h_{\infty}^{\prime}\left(r_{*}\right)$, from (43) for small $\varepsilon>0$ and for all large $\lambda>0$ it follows that there exists a unique intersection of the functions $u_{0}(r)$ and $U(r ; \lambda)$ in the connected interval $\left\{r>0|| u_{0}(r) / h_{\infty}(r)-1<\varepsilon\right\}$. Then by l.emma 2 (ML) is valid.

(ii) See the proof of (iii) of Theorem 1.

(iii) See the proof of (iv) of Theorem 3 . 


\section{References}

[1] S. ANGENENT: The zero set of a solution of a parabolic equation. I, reine angew. Math., 390, 78-96 (1988).

[2] A. FRIEDMAN: On the regularity of the solutions of nontinear elliphic and parabolic sistems of partial differential equations, J. Math. Mech.. 7, 43-59 (1958).

[3] A. Friedoman: Partial Differential Equations of Parabolic Typo. PrenticeHall. Englewood Cliffs, New Jersey (1964).

[4] V. A. GAI.AKTIONOV: On conditions of localization of unbounded solutions of quasilineat parabolic equations. Doklady AN SSSR, 264, 1035-1040 (1982) (in Russian).

[5] V. A. Gainktionor, S. P. Kurdyumov and A. A. Samarskil: On struture of limising distributions for b/on-up solutions of quasilinear parabolic 'quations. Doklady AN SSSR. 301.781-785 (1988) (in Russian).

[6] V. A. GALAKTIONOV and S. A. POSASHKOV: Applications of ne' comparison therems for tmbounded solutions of wonlinear parabolic equations. Differentzial'nye Uravnenia, 22, 1165-1173 (1986) (in Russian).

[7] V. A. GAlAKlonov and S. A. POSAsikov: Ant large solution of nomlinear heat estaduction equation with source becomes monofome in time. P'reprint Inst. Appl. Math. Acad. Sci. USSR. n.o 109 (1989) (in Russian).

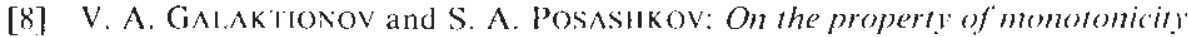
for quasilinear degenerafe parabolic equation. Differentzial'nye Uravnenia, 26. $1127-1136$ (1990) (in Russian).

[9] Y. Gilis and R. V. KOrN: Chararerizing blow-lup using similarify varahles, Indiana Univ, Math. J.. 36, 1-40 (1987).

[10] D. D. JOSEPH and T. S. I.UNDGREN: Quasilinear Dirichlet problems driven bl. posisive sources, Arch. Rational Mech. Anal., 60, 171-183 (1976).

[11] A. S. KAlAsunikov: Some questions of qualiative theor: of nominear degenerate parabolic equarions of the second order. Uspekhi Matem. Naluk. 42. 135-176 (1987) (in Russian).

[12] C. I.OEWNER and L. NIRENBI:Rg: Partal differental equations invarian under. confomal and projecrive tratnsformations. In: Contributions to Analysis. Academic Press. New York. 245-272 (1974).

[1.3] H. MATAvo: Nonincrease of the lap namber of a solution for a one-dimensional xemi-linear parabolic equation, J. Fac. Sci. Univ. Tokyo. Sec. 1A. 29, $401-441(1982)$.

[14] K. Nick1:1: Gestallaussagen wher Lostutgen Parabolischer Differenzialgheichunge't, J, reine angew. Math.. 2/1.78-94 (1962).

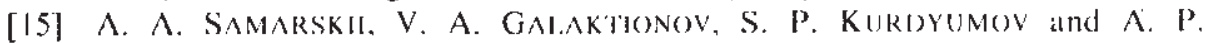

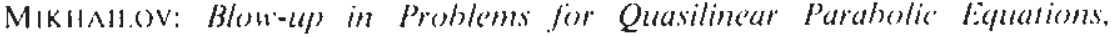
Nalukal. Moscow (1987) (in Russian).

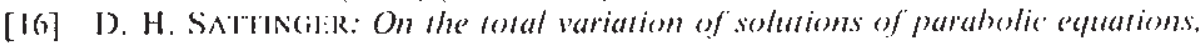
Math. Ann.. /8.3, 78-92 (1969). 